\title{
O ATENDIMENTO PSICOPEDAGÓGICO HOSPITALAR E AS TECNOLOGIAS ASSISTIVAS: IMPORTANTES ALIADOS NO PROCESSO DE INCLUSÃO
}

\section{HOSPITAL PSYCHOPEDAGOGICAL CARE AND ASSISTIVE TECHNOLOGIES: IMPORTANT ALLIES IN THE INCLUSION PROCESS}

\author{
Kathy Souza Xavier de Araújo* \\ ORCID: https://orcid.org/0000-0002-8631-3731 \\ Klebson Felismino Bernardo** \\ ORCID: https://orcid.org/0000-0002-9195-9844 \\ Janine Marta Coelho Rodrigues*** \\ ORCID: http://orcid.org/0000-0002-9457-9070
}

\section{Resumo}

$\mathrm{O}$ artigo corresponde a um relato de experiência psicopedagógica transformadora com a utilização de tecnologias assistivas numa classe hospitalar do projeto de extensão PROBEX-UFPB "Atendimento psicopedagógico à criança e ao adolescente hospitalizado", projeto realizado desde 2001 no Hospital Universitário Lauro Wanderley (HULW), localizado na cidade de João Pessoa - PB. O objetivo é reconhecer o uso das tecnologias assistivas no atendimento psicopedagógico, assim como de práticas pedagógicas inclusivas e sua relevância para a autonomia e o desenvolvimento cognitivo de alunos/as pacientes. A metodologia envolveu pesquisa bibliográfica e observacional, construída por meio de uma abordagem qualitativa, tendo como método de análise a análise de conteúdo. Constata-se, através da observação, que as tecnologias na educação de crianças e adolescentes hospitalizados possibilitaram condições mais oportunas de aprendizado e sua importância dentro da educação e o quanto é necessário lutar pela educação, que é de todos/as.

Palavras-chave: Atendimento psicopedagógico; Tecnologias assistivas; Classe hospitalar; Alunos-pacientes.

\begin{abstract}
This paper is an account of a transformative psychopedagogical experience with the use of assistive technologies in a hospital class that is part of the PROBEX-UFPB extension project "Psychopedagogical assistance to hospitalized children and adolescents". The project has been carried out since 2001 at the Lauro Wanderley University Hospital (HULW), located in the city of João Pessoa - PB. The article aims to recognize the use of assistive technologies in psychopedagogical care, as well as inclusive pedagogical practices and their relevance for the autonomy and cognitive development of students/patients. The methodology joined bibliographic and observational research, built through a qualitative approach, using content analysis as the method of analysis. The observation revealed that technologies employed in the education of hospitalized children and adolescents have enabled proper conditions for learning, it also highlighted their importance within education and how much we need to fight for more inclusive education.
\end{abstract}

Keywords: Psychopedagogical assistance; Assistive technologies; Hospital class; Student-patients. 


\section{Introdução}

Buscou-se reconhecer o uso das tecnologias assistivas no atendimento psicopedagógico, assim como de práticas pedagógicas inclusivas e sua relevância para a autonomia e o desenvolvimento cognitivo de alunos/as/pacientes, por meio de uma experiência psicopedagógica educativa desenvolvida no projeto de extensão "Atendimento psicopedagógico à criança e ao adolescente hospitalizado", da Universidade Federal da Paraíba - UFPB, realizado numa classe hospitalar do Hospital Universitário Lauro Wanderley (HULW), na cidade de João Pessoa - PB.

Este projeto está vinculado aos programas do Centro de Educação - CE e ao Programa de Pós-Graduação em Educação - PPGE da Universidade Federal da Paraíba. Desde a sua criação, em 2001, o referido projeto de extensão desenvolve atividades inovadoras no setor de pediatria do HULW, num espaço psicopedagógico que trabalha a escolarização, resgatando a autoestima das crianças e adolescentes hospitalizados.

As atividades de extensão são realizadas por bolsistas e voluntários, sendo estagiários(as) do curso de Pedagogia, Psicopedagogia e alunos(as) voluntários(as) de diversas licenciaturas, mestrado e doutorado. Neste espaço, são desenvolvidas atividades de escolarização, chamadas pelos internos de "escolinha do hospital", com atividades diversificadas também para os acompanhantes, objetivando oferecer situações de melhoria do estado de saúde e a quebra da rotina hospitalar aos envolvidos, bem como um espaço alternativo de trabalho e aprendizagem aos alunos universitários, num ambiente não formal de educação.

Através do planejamento e execução das atividades pedagógicas e psicopedagógicas, vivenciam-se diversas atividades e oficinas contextualizadas, onde se considera a prática social do sujeito e o seu contexto nas atividades que serão aplicadas. Busca-se o seu protagonismo, um maior interesse e entendimento do conteúdo trabalhado. Para tal, são criadas situações comuns ao dia a dia do aluno(a)/paciente, fazendo-o(a) interagir ativamente, de modo intelectual e afetivo, trazendo o cotidiano para o ambiente educativo e aproximando as suas rotinas do conhecimento científico.

Em um caderno de campo, registram-se comportamentos e eventos que, ao final de cada etapa do projeto, acrescentando os dados dos relatórios parciais, comporão material de discussões e publicações. E no campo de estágio, volta-se ao ensino e à pesquisa para composição de TCC de graduação, monografias de especialização, dissertações de mestrado, teses de doutorado e artigos, que são publicados com frequência.

Este projeto de extensão é de suma importância para a comunidade acadêmica e para a sociedade paraibana, pois vem fazendo a diferença na vida de muitas crianças e adolescentes hospitalizados e suas famílias, assim como da vida e prática acadêmica de muitos estudantes que passam por tal experiência. Corrobora, portanto, durante 20 anos de existência, de forma incisiva com o princípio da indissociabilidade, no que se refere à pesquisa, ensino e extensão, como eixo de ação e reflexão universitária. 
Atualmente, cada vez mais os recursos de tecnologias assistivas têm sido utilizados na educação especial, sendo pertinente pensar a possibilidade de transformar um ambiente tão hostil, como o hospital, no acesso à educação, assegurando aos(às) alunos(as) pacientes uma aprendizagem expressiva que corresponda à sua realidade, de modo a transformar e melhorar a sua qualidade de vida.

O texto apresenta algumas discussões pertinentes à luz de alguns teóricos que tratam do tema e que permeiam a psicopedagogia no ambiente hospitalar e a inclusão no contexto das classes hospitalares, assim como a descrição de atividades observadas durante a pesquisa. Verificaram-se algumas atividades realizadas com o uso de tecnologias assistivas no atendimento psicopedagógico e os reais impactos na vida de crianças e adolescentes hospitalizados por tempo prolongado, de modo a se discutir acerca da relevância de desenvolver atividades inclusivas visando tornar o ensino/aprendizagem cada vez mais lúdico e atraente.

Desta forma, possibilita-se aos estudantes e professores o desenvolvimento de tarefas contextualizadas e interessantes, porém, respeitando as reais limitações do(a) aluno(a) paciente e suas necessidades de aprendizagem, além de caracterizar onde este atendimento educacional pode acontecer. Por outro lado, demonstra-se, neste estudo, a forma como foram trabalhadas essas tecnologias com crianças e adolescentes atendidos/as, para que estes/as estivessem integrados/as com os acontecimentos externos e conectados/as com temas atuais, buscando essa educação de forma global e integral.

A proposta metodológica deste estudo envolveu pesquisa bibliográfica, com aporte de trabalhos acadêmicos, livros e documentos oficiais, assim como a observação realizada. Segundo Gil (2010), a pesquisa bibliográfica é elaborada com base em material já publicado e tem o objetivo de analisar posições diversas em relação a determinado assunto. Buscou-se, dessa forma, um aprofundamento do objeto de pesquisa e de trabalhos desenvolvidos nesse âmbito.

A pesquisa foi construída na perspectiva qualitativa de Minayo (2014), onde a pesquisa qualitativa responde a questões muito particulares e se preocupa, nas ciências sociais, com um nível de realidade que não pode ser quantificado. A técnica de observação participante se realiza através do contato direto do pesquisador com o fenômeno observado, para se obterem informações sobre a realidade dos atores sociais em seus próprios contextos. O observador, enquanto parte do contexto de observação, estabelece uma relação face a face com os observados (MINAYO, 2002).

Assim, é desenvolvida uma ótica psicossocial, ancorada nas bases da fenomenologia de Husserl (2001), segundo o qual

A análise intencional deixa-se guiar por uma evidência fundamental: todo o cogito, enquanto consciência é, num sentido muito largo, significação da coisa que visa, mas esta significação ultrapassa a todo instante aquilo que, no próprio instante, é dado como explicitamente visado. Ultrapassa-o, quer dizer, é maior com um excesso que se estende para o além (HUSSERL, 2001, p. 65). 
A fenomenologia de Edmund Husserl considera, do ponto de vista metodológico, a suspensão do juízo em relação à posição de existência do mundo, para recuperá-lo, na consciência, de maneira incontestável, no seu puro significado. Se a adoção do programa positivista nas ciências humanas limita-nos a uma lógica indutiva e probabilística, o método fenomenológico nas ciências humanas convida-nos a exercer uma atitude reflexiva e analítica acerca do que há de mais originário na coisa sobre a qual retornamos.

Utilizando a análise do conteúdo, com o aporte de Minayo (2002), a análise de conteúdo é "compreendida muito mais como um conjunto de técnicas", possibilitando uma aplicação bastante variada, com duas funções: verificação de hipóteses e/ou questões e descoberta do que está por trás dos conteúdos manifestos. Tais funções podem ser complementares, com aplicação tanto em pesquisas qualitativas como quantitativas. Percorre-se, assim, um caminho crítico e reflexivo frente aos desafios enfrentados pelos envolvidos com a psicopedagogia hospitalar.

Há, sobretudo, relevância social, pois a proposta desta pesquisa é fundamentada para contribuir no âmbito psicopedagógico e educacional, promovendo discussões e divulgações a respeito das contribuições e reais impactos do uso das tecnologias assistivas no processo psicopedagógico/educacional desenvolvido no contexto hospitalar.

\section{Tecnologias assistivas e práticas psicopedagógicas}

Com as transformações e os avanços ocorridos na sociedade, cresce o interesse por estudos acerca das Tecnologias Assistivas, considerada cada vez mais como um recurso extremamente relevante para a autonomia e inclusão escolar e social. A Tecnologia Assistiva é diferente da tecnologia reabilitadora, usada, por exemplo, para auxiliar na recuperação de movimentos diminuídos.

O conceito de Tecnologia Assistiva é diferente das tecnologias de reabilitação, por estarem diretamente ligadas a recursos que atendem as necessidades diretas do indivíduo, visando sua independência e autonomia. Já os recursos de reabilitação visam o diagnóstico ou tratamento na área da saúde, sendo, portanto, recursos de trabalho dos profissionais dessa área. No Brasil, a TA é definida como:

Tecnologia assistiva é uma área do conhecimento, de característica interdisciplinar, que engloba produtos, recursos, metodologias, estratégias, práticas e serviços que objetivam promover a funcionalidade, relacionada à atividade e participação, de pessoas com deficiência, incapacidades ou mobilidade reduzida, visando sua autonomia, independência, qualidade de vida e inclusão social (BRASIL, 2007, p. 3). 
As Tecnologias Assistivas vêm tornando-se, cada vez mais, uma ponte para abertura de novas oportunidades nos processos de aprendizagem e desenvolvimento de práticas pedagógicas inclusivas. Bresch (2008) define Tecnologia Assistiva como:

\begin{abstract}
A TA deve ser entendida como o "recurso do usuário" e não como "recurso do profissional". Isto se justifica pelo fato de que ela serve à pessoa com deficiência que necessita desempenhar funções do cotidiano de forma independente. Por exemplo: a bengala é da pessoa cega ou daquela que precisa de um apoio para a locomoção; a cadeira de rodas é de quem possui uma deficiência física e com este recurso chega aos lugares que necessita; a lente servirá a quem precisa melhorar sua eficiência visual. O software leitor, fala o conteúdo de textos digitalizados à pessoa com deficiência visual ou a quem não consegue ler em função da dislexia ou deficiência intelectual. Todos estes recursos promovem maior eficiência e autonomia nas várias atividades de interesse de seus usuários. Por princípio, o recurso de TA acompanha naturalmente o usuário que o utilizará em diferentes espaços na sua vida cotidiana (BERSCH, 2008, p. 11).
\end{abstract}

Estudos e pesquisas têm revelado que, apesar das Tecnologias Assistivas serem elementos espetaculares para a educação, por outro lado infelizmente nos deparamos com uma complexa realidade, com diversos fatores que dificultam a apropriação desses recursos para a inclusão educacional, principalmente com as contradições e incongruências existentes no nosso contexto escolar. $\mathrm{O}$ atendimento psicopedagógico/educacional no ambiente hospitalar é um espaço diferente, que ultrapassa os espaços escolares e que diverge de toda estrutura e recursos que uma instituição comum possa oferecer. A educação nesse ambiente vem carregada de outros elementos, principalmente emocionais, como medo e insegurança, assim como fragilidades causadas pelas enfermidades em que foram acometidos/as estes/as alunos/as/pacientes.

De acordo com Rodrigues (2012), a educação tem que ir até onde se faz necessária, sendo o ambiente hospitalar um desses lugares, e o profissional da educação precisa estar preparado para atuar nesses espaços. Se existem espaços pedagógicos além da escola, é sinal de que se faz necessário a presença de profissionais que atuem de forma diversificada. Atualmente, existe um amplo debate nacional e internacional acerca das práticas inclusivas que se constituem um desafio político-pedagógico, porém, quando entramos na esfera de classes hospitalares, este debate torna-se bastante sutil, sendo um grande desafio para os profissionais envolvidos e para as crianças e adolescentes hospitalizados.

Desta forma, o apoio de um psicopedagogo/a é primordial para o processo, pois sua contribuição pode ajudar a romper barreiras, fazendo com que estes/as alunos/as/pacientes resgatem a autoestima e sintam-se respeitados e acolhidos. Trata-se de um momento difícil, em que se encontram num estado de desânimo e insegurança, vindo o/a psicopedagogo/a com a aplicação de atividades educativas, estimulando a criação, a leitura, a socialização, através da ludicidade, contribuindo na promoção da saúde e sensação de bem-estar. 
$\mathrm{O}$ art. 74 da lei $\mathrm{N}^{\mathrm{o}}$ 13.146, de 6 de julho de 2015, que trata da tecnologia assistiva na inclusão da "Pessoa com Deficiência", no seu Art. 74 diz: "É garantido à pessoa com deficiência acesso a produtos, recursos, estratégias, práticas, processos, métodos e serviços de tecnologia assistiva que maximizem sua autonomia, mobilidade pessoal e qualidade de vida" (BRASIL, 2015, p. 54). Deste modo, corrobora-se a necessidade de construirmos cada vez mais sistemas educacionais inclusivos, valorizando e proporcionando a crianças e adolescentes a garantia à saúde, à educação e à dignidade humana.

Segundo Mattos e Mugiatti (2009, p. 41), "Todas as crianças têm direito ao ensino escolar; mas para isso é necessário criar espaço de ensino nos hospitais pediátricos, ou correlatos, onde estejam hospitalizados crianças ou adolescentes em idade de escolarização". Faz-se necessário, então, a reflexão acerca do possível caminho para encorajá-los/as a seguir em frente, oportunizando práticas pedagógicas inclusivas em classes hospitalares, de modo a evitar, assim, situações de isolamento. Cada experiência (positiva ou negativa) vivenciada no período de hospitalização traz consequências.

A utilização das tecnologias assistivas vem justamente nessa perspectiva, não só de apenas garantir o ensino no ambiente hospitalar, mas também de facilitar o ensino/ aprendizagem destas crianças e adolescentes, tornando o aprendizado mais tranquilo e composto de uma carga de ludicidade.

\section{Uma experiência educativa transformadora}

$\mathrm{Na}$ classe hospitalar, deve-se usufruir de meios educativos variados e que promovam o envolvimento e desenvolvimento do educando/paciente com o conhecimento fora do ambiente escolar. Tais atividades devem envolver atividades lúdicas, que promovam distração e diversão ao hospitalizado. É preciso criar um ambiente de ensino no qual os/as hospitalizados/as com idade de escolarização possam frequentar, de acordo com a realidade da situação de sua saúde, transformando o ambiente em que eles/as se encontram em ambiente menos hostil, onde o psicopedagogo tem um papel determinante na condução desta aprendizagem, pois, segundo Cunha (2014), a aprendizagem criativa é uma experiência consciente, manipulada e transformadora.

Sob esta ótica, inserir as Tecnologias Digitais de Informação e Comunicação na educação de crianças e adolescentes hospitalizadas é um meio que possibilita condições mais oportunas de aprendizado, principalmente dos/as estudantes que possuem alguma forma de limitação motora e/ou cognitiva, como é o caso aqui descrito. Foram verificadas algumas atividades que possibilitaram o processo de ensino-aprendizagem nos atendimentos psicopedagógicos/educacionais. Em um dos atendimentos psicopedagógicos, foi utilizado um acessório simulador de holograma, juntamente com um celular como recursos facilitadores, o que proporcionou um momento de aprendizagem muito mais atraente e que despertou a curiosidade dos/as educandos/as. 
Figura 1 - Modelo da aula aplicada com Holograma

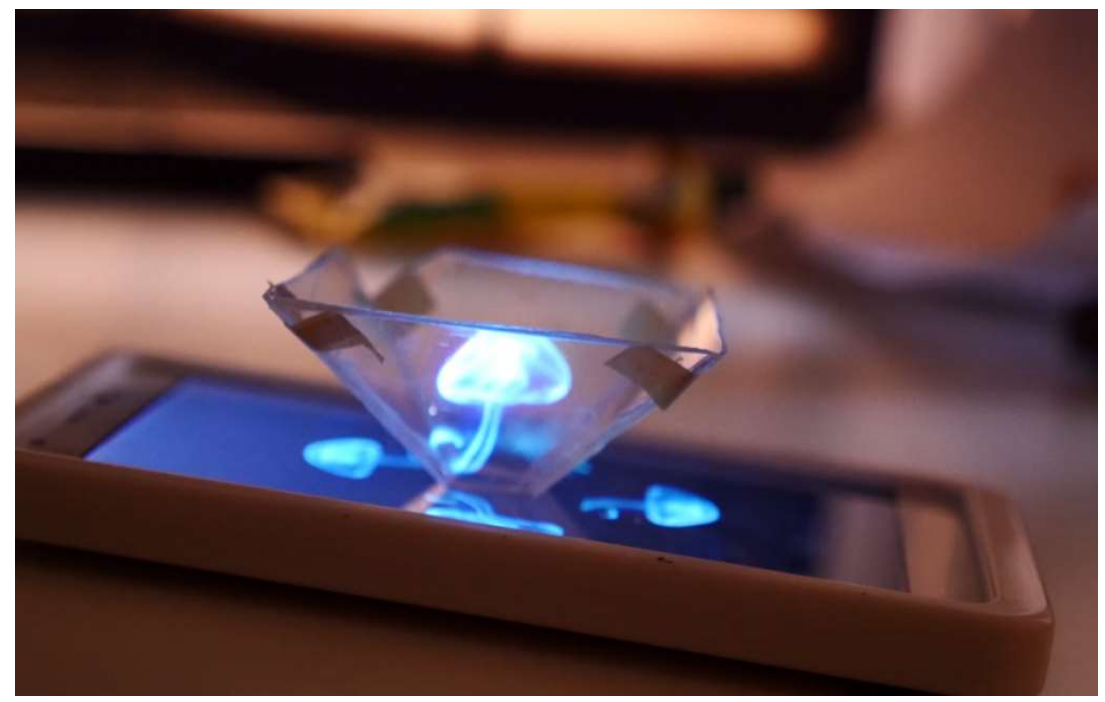

Fonte: RAMOS, 2020.

O presente trabalho apresenta uma proposta de atendimento psicopedagógico diferenciado para classes hospitalares. Pensou-se em várias estratégias de ensino que facilitasse e possibilitasse um estreitamento maior com o conhecimento, porém, a limitação de alguns/as alunos/as pacientes, principalmente motora, era um grande empecilho, mas não impossível. Nesse contexto, a ludicidade é sempre bem-vinda, sendo um elemento transformador das classes hospitalares, baseado no brincar e no aprender de uma forma mais divertida e agradável.

Segundo Rodrigues (2012, p.93), "as brincadeiras, atenção e carinho fazem parte das atividades otimizadoras adotadas pelos professores das classes hospitalares para auxiliar os alunos-pacientes a recuperarem a saúde e a autoestima". Dessa forma, acredita-se que as estratégias de ensino diferenciadas podem facilitar a ação do psicopedagogo na escolarização de crianças e adolescentes hospitalizados, bem como o profissional deve estar atento às especificidades de cada aluno/paciente, para aplicar a melhor estratégia de ensino, utilizando a metodologia e a tecnologia assistiva mais adequada para cada caso.

Outra atividade observada e com resultado positivo foi a utilização dos óculos de realidade virtual com uma aluna/paciente acometida de atrofia muscular, porém, com seu cognitivo $100 \%$ preservado. Uma vez que ela ficou impossibilitada de manusear qualquer objeto, este recurso facilitou os momentos de aprendizado e as atividades recreativas.

A caixa VR óculos 3D utilizada foi de dimensões 180 x 120 x $100 \mathrm{~mm}$, podendo ser usado para telefone celular, Tablet PC, MID, TV Box, PC, gamepad, mouse sem fio, portátil Bluetooth Selfie remoto para celular e computador com função TV Box remoto, GW: 0,52 
$\mathrm{kg}$, e controlador remoto Bluetooth (tanto para iOS e Android), o que veio a facilitar o manuseio e as configurações durante as atividades.

Desta forma, por meio de um plano de atividades previamente elaborado (sequência didáticas), associado à utilização das Tecnologias da Informação e Comunicação (TIC's) como Tecnologias Assistivas, possibilitou-se aos/as alunos/as mais autonomia e favorecimento para o desenvolvimento de sua metacognição. Ressaltamos que isso ocorreu dentro das limitações de cada um e da exploração da criatividade, onde posteriormente foram apresentados e oferecidos novos recursos pedagógicos e tecnológicos, tornando, assim, possível a inclusão digital, social e escolar dos/as mesmos/as.

Diante das limitações causadas pela doença, foram criadas oportunidades de aprendizagem que favorecessem a estes/as educandos/as romper as barreiras que dificultam o seu acesso ao saber. E um dos meios simples e de pouco custo e acessível foi uso das Tecnologias, desde o simples uso de uma TV até o uso dos óculos de realidade virtual, ou seja, a exploração desses recursos veio como um suporte de apoio na escolarização dos/as alunos/as/pacientes em questão.

A presença do psicopedagogo/a nesses espaços pedagógicos não formais e de aprendizagem humana é imprescindível, pois como afirma Porto (2008):

\footnotetext{
Considerando que a proposta da psicopedagia hospitalar é ser o interlocutor não só das crianças e adolescentes, mas também de todos aqueles que passam por internações, sejam elas curtas, médias e de longas durações, doenças crônicas e de pacientes terminais, dando o de melhor de nossa atenção e técnica, mas criando um mundo, onde pessoas se preocupam com outras (PORTO, 2008, p. 22).
}

No que se refere a esta pesquisa, pudemos constatar que durante a utilização das tecnologias assistivas os/as alunos/as/pacientes demonstraram-se muito mais motivados/as e com disposição para os momentos de aprendizado. Estes recursos vêm para ficar e facilitar o aprendizado das crianças e adolescentes hospitalizados. Abaixo, apresentamos a classificação categorial para os recursos de Tecnologia Assistiva (TA) amparados no texto da pesquisadora Rita Bersch (2008), disponível em http://www.assistiva.com.br, tendo como fonte norteadora as diretrizes gerais de uma lei norte-americana comumente conhecida como American with Disabilities Act (ADA). 
Figura 2 - Classificação categorial para os recursos de Tecnologia Assistiva (TA)

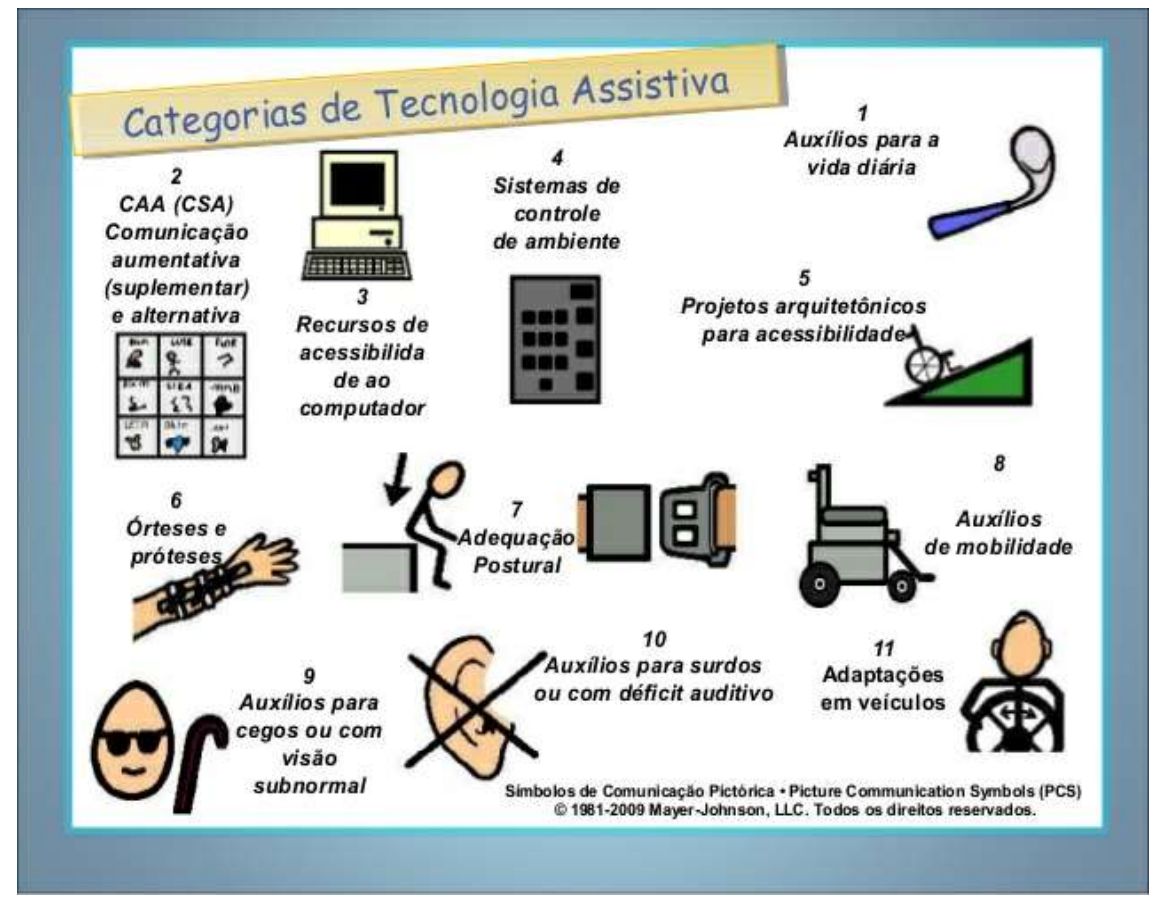

Fonte: PORTELA, 2020.

Durante este estudo, verificou-se a utilização de diversos recursos que fazem parte das categorias acima descritas, exceto as categorias 9 e 10, por não terem alunos/as/pacientes com deficiência visual e auditiva internados/as durante o período de observação. As TIC's utilizadas como TA`s observadas com mais frequência durante as atividades de extensão estão enquadradas na categoria 3. São elas: computador/notebook, celular, tablet, TV por assinatura, tecnologias de acesso remoto (Wi-Fi, Bluetooth), Websites, pendrives, cartão de memória, câmeras de vídeo, óculos de realidade virtual, dentre outras, como recursos de ao computador.

\section{Considerações finais}

Podemos concluir que, diante do que foi exposto, ficou claro que as Tecnologias da Informação e Comunicação (TIC's) sendo utilizadas como Tecnologias Assistivas e como ferramenta no atendimento psicopedagógico possibilitaram aos alunos/as/pacientes alcançar o desenvolvimento de habilidades e aprendizagens que lhes ajudaram a ter mais autonomia. Estes recursos deixam de ser apenas aparelhos tecnológicos de entretenimento, passando a 
ser um material pedagógico/educativo muito mais atrativo e versátil, auxiliando, dentro de uma perspectiva da mediação da aprendizagem e dentro de uma visão da educação inclusiva, incrementando uma proposta de ensino-aprendizagem mais ativa tanto de quem ensina como também de quem aprende.

As Tecnologias Assistivas utilizadas destacaram-se como mais um recurso de acessibilidade ao saber (currículo), minimizando as limitações evidentes e existentes em cada caso específico, garantindo aos alunos/as/pacientes o acesso ao saber com a utilização de práticas educativas inovadoras, vindo a romper barreiras práticas e intelectuais para a construção da sua autoconfiança e da certeza de que são capazes de ir além. Percebemos que as Tecnologias na educação de crianças e adolescentes hospitalizados possibilitaram condições mais oportunas de aprendizado, vendo que os/as alunos/as/pacientes possuíam alguma forma de limitação motora e/ou cognitiva.

Foram explorados meios de prender a atenção desses/as alunos/as, os quais facilmente se dispersam, devido à sua condição de adoecimento, perdendo facilmente o interesse. Foram aplicadas atividades interessantes, dinâmicas e criativas, com vistas a fugir do método tradicional de ensino. Isso tornou os momentos de aprendizagem mais agradáveis e significativos, adicionados de interações afetivas, visto que o aluno/a/paciente se encontra impossibilitado/a de frequentar a escola regular e o seu ambiente social, o que promove, na maioria das vezes, uma baixa na sua autoestima.

As Tecnologias da Informação e Comunicação (TIC's), utilizadas como Tecnologias Assistivas, ainda representam um tema que precisa ser mais debatido e discutido no âmbito das políticas públicas de inclusão, de forma que busque proporcionar a equidade escolar e social, o rompimento das barreiras e diferenças, criando outras possibilidades e a participação do sujeito na sociedade. Além disso, de ajudar na autonomia do/a aluno/a-paciente, o que reflete diretamente na sua autoestima e no sentimento de pertencimento na construção do processo educativo.

As práticas de extensão descritas neste trabalho só reforçam a importância da utilização dessas tecnologias no processo psicopedagógico hospitalar e da necessidade de as classes hospitalares adaptarem-se a essa realidade. Isso corrobora, também, a extrema importância da interação entre ensino, pesquisa e extensão. Destarte, esperamos que este estudo preliminar promova uma melhor compreensão e interesse pelo tema abordado, assim como desperte novos questionamentos do que se propõe a utilização das Tecnologias Assistivas no atendimento psicopedagógico em classes hospitalares.

Isso propicia aos/às alunos/as/pacientes um maior aprendizado, bem como preparemnos/nas para uma melhor qualidade de vida, além de colaborar para a efetiva implementação das políticas públicas de inclusão nesse processo e nos espaços educacionais. 


\section{Referências}

BERSCH, Rita. Introdução à tecnologia assistiva. v.21. Porto Alegre: CEDI, 2008.

BRASIL. Lei No 13.146, de 6 de julho de 2015. Institui a Lei Brasileira de Inclusão da Pessoa com Deficiência (Estatuto da Pessoa com Deficiência). 2015. Diário Oficial da União. Disponível em: http://www.planalto.gov.br/ccivil_03/_Ato20152018/2015/Lei/L13146.htm. Acesso em: 01 jun. 2020.

BRASIL. Portaria Normativa $\mathbf{n}^{\mathbf{0}}$ 13, de 24 de abril de 2007. Dispõe sobre a criação do Programa de Implantação de Salas de Recursos Multifuncionais. Brasília, 2007.

CUNHA, Eugênio. Práticas pedagógicas para inclusão e diversidade. 4. ed. Rio de Janeiro: Wak Editora, 2014.

GIL, Antônio Carlos. Como elaborar projetos de pesquisa. 5. ed. São Paulo: Atlas, 2010.

HUSSERL, Edmund. Meditações cartesianas. São Paulo: Madras, 2001.

MATOS, Elisete Lúcia Moreira; MUGIATTI, Margarida Maria Teixeira de Freitas.

Pedagogia Hospitalar: a humanização integrando educação e saúde. Petrópolis, RJ: Vozes, 2009.

MINAYO, Maria Cecília de Souza (org.). Pesquisa Social: Teoria, método e criatividade. 21. ed. Petrópolis: Vozes, 2002.

MINAYO, Maria Cecília de Souza. O desafio do conhecimento: pesquisa qualitativa em saúde. 14. ed. São Paulo: Hucitec, 2014.

PORTO, Olívia. Psicopedagogia Hospitalar: intermediando a humanização na saúde. Rio de Janeiro: Wak Ed, 2008.

PORTELA, Regina Lucia. Nem bem no céu... Nem bem na terra: O mercado da tecnologia assistiva. 2 jul. 2017. Online. Disponível em:

https://reginaluciaportela.blogspot.com/search?q=categorias+TA. Acesso em: 15 set. 2020.

RAMOS, Ademilson. Aprenda a fazer um holograma 3D com seu smartphone. 29 set. 2020. Disponível em: https://engenhariae.com.br/tecnologia/aprenda-a-fazer-umholograma. Acesso em: 15 set. 2020.

RODRIGUES, Janine Marta Coelho. Classes Hospitalares: espaço pedagógico nas unidades de saúde. Rio de Janeiro: Wak Ed, 2012. 